Relato de experiencia profesional

\title{
Musicoterapia en la estrategia de atención primaria de la salud en el marco de la pandemia por Covid-19: Experiencia en un hospital rural
}

\section{Carrizo, Lucia}

\author{
Lucia Carrizo \\ luciajcarrizo@gmail.com
}

\author{
ECOS - Revista Científica de Musicoterapia y \\ Disciplinas Afines \\ Universidad Nacional de La Plata, Argentina \\ ISSN-e: 2718-6199 \\ Periodicidad: Semestral \\ vol. 6, núm. Esp.3, e009, 2021 \\ revista.ecos@presi.unlp.edu.ar \\ Recepción: 07 Junio 2021 \\ Aprobación: 06 Octubre 2021 \\ URL: http://portal.amelica.org/ameli/ \\ jatsRepo/459/4592620004/index.htm \\ DOI: https://doi.org/10.24215/27186199e009
}

\section{c) (i) $\Theta$}

Esta obra está bajo una Licencia Creative Commons AtribuciónNoComercial-SinDerivar 4.0 Internacional.
Resumen: El presente trabajo tiene como objetivo dar cuenta de la inclusión de la musicoterapia en la estrategia de Atención Primaria de la Salud (en adelante APS) a partir de una experiencia de rotación de cuatro meses por el Hospital Rural de Trevelin. Ésta se dio en el marco de la formación de postgrado de la Residencia Interdisciplinaria en Salud Mental Comunitaria (en adelante RISMC) de la localidad de Esquel, provincia de Chubut, Argentina, durante los meses de octubre de 2020 hasta enero de 2021 inclusive. Se han seleccionado para los fines de este artículo aquellas intervenciones que permiten ilustrar mejor la inclusión como musicoterapeuta en salud mental comunitaria ligadas a la prevención primaria y promoción de salud, en el marco de la pandemia por Covid-19. Se describirán para esto las experiencias de trabajo conjunto con lxs trabajadores comunitarios de salud en terreno (en adelante TCST), consultorios compartido con pediatría y otras experiencias con el equipo de salud que permitirán describir algunos de los aspectos fundamentales de la Estrategia de Atención Primaria.

Palabras clave: Musicoterapia en Salud Mental Comunitaria, Atención Primaria de la Salud, COVID 19, prácticas territoriales.

Abstract: The present work aims to account for the inclusion of music therapy in the strategy of Primary Health Care (hereinafter PHC) from a four-month rotation experience at the Rural Hospital of Trevelin. This was given within the framework of the postgraduate training of the Interdisciplinary Residence in Community Mental Health (hereinafter RISMC) in the town of Esquel, Chubut province, Argentina, during the months of October 2020 to January 2021 inclusive. For the purposes of this article, the interventions that better illustrate the inclusion as a music therapist in community mental health related to primary prevention and health promotion in the framework of the COVID-19 pandemic have been selected. This experiences shared with community health workers (hereinafter TCST), pediatricians and the health team from the hospital allows to build some of fundamentals aspects and strategies in primary Healthcare.

Keywords: Music Therapy in Community Mental Health, Primary Health Care, COVID 19, territorial practices. 
"y me llega latente y agresiva la visión del canal que lo atraviesa cinta de agua que nace

Percey arriba y en sus calles sutil se despereza"

Fuente: (Pazos Rios, R. Y Grupo vocal Sur, 1989)

\section{INTRODUCCIÓN}

La experiencia que se relata a continuación tuvo lugar en la localidad cordillerana de Trevelin. La misma está situada al oeste de la provincia de Chubut, República Argentina. A nivel demográfico la proyección de población para el año 2020 fue de 9520 personas (Dirección General de Estadística y Censos, s. f.) Su paisaje cordillerano se combina con zonas de campos y el acceso a la costanera del río permite un fácil acercamiento a la naturaleza. Dentro de la población local hay descendientes de la comunidad galesa, como también de pueblos originarios mapuche-tehuelche, siendo esto un dato significativo a nivel cultural e histórico.

El Hospital Rural de Trevelin cuenta con pediatría, clínica médica y medicina general, internación y guardia, salud mental, rehabilitación, trabajadores comunitarios de salud en terreno (en adelante TCST), enfermería, vacunatorio, laboratorio, rayos y odontología. Del mismo nosocomio dependen 4 parajes rurales (Lago Rosario-Sierra Colorada, Los Cipreses, Aldea Escolar, Lago Futalaufquen) y un centro de atención primaria de la salud, ubicado en uno de los barrios. A su vez cuenta con dos dispositivos ocupacionales/ laborales (Ministerio de Salud de la Provincia de Chubut, 2016) "El Caldero" y "Vive la huerta". El equipo de salud mental está conformado por psicología, trabajo social, terapia ocupacional y operadores, pudiendo así facilitar el acceso a tratamientos y a diferentes opciones terapéuticas.

El trabajo en hospitales rurales, centros de atención primaria y puestos sanitarios, se encuentra fundamentado en la Estrategia de Atención Primaria de la salud (APS) la cual propicia la asistencia puesta al alcance de todos los individuos y familias de la comunidad, mediante su plena participación.(OMS, 1987) Implica la descentralización del modelo hospitalocéntrico hacia uno basado en el abordaje comunitario, la delegación de funciones al trabajador comunitario de salud, participación responsable de la comunidad y coordinación con otros sectores (Argandoña, 2012) A su vez, la salud mental se incluye en la Estrategia de APS a fin de que la atención de las problemáticas psicosociales pueda brindarse en el marco comunitario y resguardando los derechos de las personas. (OPS, 1990)

La rotación se efectuó en contexto de pandemia por COVID-19 el cual necesariamente, dio lugar a una reorganización hospitalaria para evitar la acumulación de trabajadores y de pacientes en el mismo sector, teniendo en cuenta la importancia del distanciamiento para prevenir la propagación del virus. Esta circunstancia resultó un limitante en relación al armado de propuestas grupales o talleres (estrategia frecuentemente utilizada en el trabajo de salud mental comunitaria) que implicó readaptar las prácticas y crear nuevas formas de acercamiento con la comunidad.

La pandemia por Covid-19 tuvo las características de lo que se conoce como emergencias y catástrofes. "El brote de Covid-19 es un evento disruptivo, excepcional, que ha exigido reorganizar la vida de todos, a la vez que genera incertidumbre en relación al futuro y esto lo transforma en amenazante." (Dirección Nacional de Salud Mental y Adicciones [DNSMyA], 2021) Esto sumado a las consecuencias que podían tener las medidas de distanciamiento en la subjetividad y en el lazo social hacía necesario el abordaje en salud mental a toda la población. A su vez, las consecuencias de las medidas preventivas tuvieron un impacto socioeconómico para muchas personas de la localidad ya que muchas familias que viven del trabajo no formal. En los parajes, la falta de transporte público que permitía el contacto con la localidad de Trevelin también fue un factor que afectó a la accesibilidad al hospital rural y otras actividades que puedan resultar significativas y generar una mayor calidad de vida para las personas. Asimismo la rotación se desarrolló en contexto de medidas de fuerza, por la crisis económica en la que el Estado Provincial adeudaba sueldos a trabajadores estatales, lo 
cual también influyó en la accesibilidad al sistema de salud. Muchas de las personas de la comunidad son trabajadores estatales y también vieron afectada su economía por esta crisis. Dada la consideración de la salud desde una perspectiva integral es necesario entender los factores sociales, económicos, condiciones de salud previas y de acceso al sistema sanitario que debían ser abordados.

La importancia del trabajo comunitario en salud mental residió en la promoción de estrategias de cuidado, solidaridad y no discriminación a fin de disminuir otros padecimientos asociados y en el favorecimiento de estrategias de afrontamiento de diversas situaciones. (DNSMyA, 2020a). Fue necesario continuar realizando estrategias de prevención primaria y promoción de salud, detectando recursos comunitarios de afrontamiento y posibles situaciones de vulnerabilidad psicosocial que se vieran dificultadas aún más por las medidas de restricción de la pandemia.(DNSMyA, 2020b) El abordaje territorial fue necesario y la inclusión de trabajadores de salud mental en la estrategia de atención primaria de la salud formó parte de la respuesta para la asistencia en el contexto de emergencia sanitaria. (DNSMyA, 2020b)

Se describirán a continuación las experiencias desarrolladas en el hospital que permiten profundizar en características de la APS: participación de las personas en los procesos de salud, accesibilidad al sistema de salud, delegación de funciones en lxs TCST, promoción de la salud y prevención primaria.

\section{Desarrollo DE LA EXPERIENCIA}

La atención primaria de la salud se lanzó en 1978 en la Conferencia de Alma-Ata, como "un sistema de valores, principios y enfoques destinados a mejorar el grado de salud de las poblaciones desfavorecidas." (Argandoña, 2012, p. 7) Fue una forma de descentralizar la atención hospitalocéntrica en la que la prevención es un factor importante.

La participación de las personas y comunidades en su propio proceso de salud es uno de los aspectos fundamentales de la APS. Para esto es necesario escuchar a la comunidad en la descripción de sus necesidades, problemas y fortalezas. En un trabajo anterior (Carrizo, 2019) se describió la metodología del diagnóstico participativo comunitario utilizada dentro del equipo de la RISMC. Al adentrarse en una nueva comunidad, fue necesario poder conocer a la misma teniendo en cuenta la percepción de las personas implicadas, para lo cual se tuvo en cuenta la metodología del diagnóstico participativo comunitario, adaptándola a la situación de covid-19 y al corto tiempo destinado a la rotación.

Dicho diagnóstico cuenta con herramientas específicas como entrevistas informales a trabajadores de salud, entrevistas abiertas a los pobladores, cartografía social, talleres diagnósticos, etc. Desde la musicoterapia, este diagnóstico se realiza desde la sensibilización y escucha de los entornos sonoros y de la estética comunitaria, proponiendo experiencias musicales participativas, que permiten conocer diferentes aspectos. históricos, culturales, afectivos, cognitivos, sociales de las comunidades. "La estética comunitaria mira, escucha y recrea los modos de moverse de la sociedad: prisas pausas, retardos, distancias, rumbos, emplazamientos, concentraciones, lugares, usos, abusos, desusos, cantidades, colores, tamaños, intensidades, volúmenes, precios, ruidos, silencios, sonidos, texturas, suavidades, durezas, rupturas, etc. " (Christlieb en Pellizari, 2011, p. 58)

Estos aspectos se consideran importantes a la hora de pensar determinantes de salud, entendiendo a la misma desde una perspectiva integral. A partir de la escucha de las formas sensibles se indaga en el sentido de vida de las personas, actividades de interés, vínculos, creencias, sueños, a la vez que se perciben las modalidades de construcción de salud en la vida cotidiana de las personas que habitan cierta comunidad (Pellizari, 2011). Es importante recordar la importancia que tiene en el contexto de pandemia por Covid 19 y para la estrategia de APS, conocer las estrategias saludables de una comunidad para afrontar diversas situaciones. (DNSMyA, 2020b)

"Si la salud es no solamente la ausencia de afecciones y enfermedades, es preciso reorientar tales sistemas para llegar a toda la población y promover su bienestar físico, mental y social. La estrategia de la Atención Primaria de Salud (APS), 
esencial para alcanzar el objetivo de salud-bienestar para todos, enfoca las necesidades de la comunidad y utilizando sus recursos" (Argandoña, 2012, p.31).

El reconocimiento de la comunidad a partir de sus formas sensibles posibilita utilizar en las intervenciones, variables musicales que se ajusten a los tempos, ritmos, intensidades, frecuencias que son aceptables y que resulten culturalmente pertinentes para determinada comunidad, siendo este uno de los factores importantes en la estrategia de atención primaria (OMS, 1978).

\section{La atención primaria}

"Representa el primer nivel de contacto de los individuos, la familia y la comunidad con el sistema nacional de salud, llevando lo más cerca posible la atención de salud al lugar donde residen y trabajan las personas, y constituye el primer elemento de un proceso permanente de asistencia sanitaria” (OMS, 1978).

Una de las principales formas de contactar con la población de la comunidad fue la salida al territorio de forma conjunta con lxs TCST tanto urbanxs (localidad de Trevelin) como en algunos parajes. Estxs trabajadorxs realizan periódicamente visitas a distintas familias con el objetivo de censar aspectos socioambientales y problemáticas de salud (habitualmente lxs TCST visitan a familias a fin de indagar condiciones de vida habitacionales, económicas, y realizar seguimiento de personas con enfermedades crónicas, embarazo y primera infancia y adultxs mayores), muchas veces son el nexo y el vínculo de confianza entre las personas y el hospital favoreciendo la accesibilidad al mismo. Durante la pandemia de Covid 19 las visitas se realizaron en la puerta o en espacios abiertos, sin ingresar a los domicilios, manteniendo la distancia de 2 metros y con las medidas de seguridad (barbijo e higiene de manos)

Lxs TCST seleccionaban previamente a la salida algunas familias que ellxs consideraban, según su conocimiento de las mismas, para la visita conjunta con salud mental. Entre las problemáticas seleccionadas para el trabajo en conjunto se encontraban situaciones de violencia familiar, alcoholismo, dificultades en la crianza de niños, niñas y adolescentes, personas adultas mayores en situación de soledad y personas con patologías crónicas (incluyendo personas con diagnósticos de padecimiento mental) entre otras. Muchas veces la visita era el único momento posible de intervención por lo cual era importante poder identificar los aspectos de vulnerabilidad y colaborar en el armado de estrategias interdisciplinarias para la contención, si era necesario.

Durante las visitas, se propusieron experiencias musicales que facilitaron conocer y profundizar aún más diferentes aspectos de la vida de las personas. La capacidad expresiva, perceptiva y creativa y la capacidad de conectarse con una experiencia sensible como la música son indicadores aspectos saludables. Según Weistein (2011), la capacidad de goce y la creatividad forman parte de las dimensiones de salud.

Teniendo en cuenta la importancia de la participación de las personas en sus propios procesos de salud como un aspecto fundamental de la estrategia de APS. (OMS, 1978) el acompañamiento a TCST en sus visitas proponiendo experiencias musicales participativas favoreció un acercamiento diferente al habitual, propiciando que las personas visitadas pudieran posicionarse en un lugar más activo, creativo y placentero, participando de una experiencia sensible compartida de la cual eran protagonistas y no meros receptores de intervención. Asimismo la sensibilización generada a partir de experiencias estéticas, abría la posibilidad de manifestar otras temáticas en la conversación posterior, muchas veces ligadas a la identidad socio-histórica, a las capacidades y a las creencias de las personas, ampliando así los límites de la visión de la salud como mera ausencia de enfermedad y pudiendo enfocarse en los aspectos positivos que permiten crear la salud en la vida cotidiana (Pellizari, 2011).

Esta experiencia también contribuyó a que lxs TCST pudieran conocer y abordar aspectos del padecimiento mental de las personas y habilitarse a contenerlas. Por ejemplo, dar contención y una primera ayuda en salud mental a familias que transitaban momentos difíciles ya sea por casos de suicidio de un 
familiar, de consumo problemático de alcohol o de distintas afecciones de salud integral. Esto está basado en uno de los fundamentos imprescindibles de la APS, que es la delegación de funciones, el cual se refiere a la redistribución de los conocimientos, habilidades y actitudes de especialistas, siempre escasos, entre los numerosos trabajadores de APS. (Argandoña, 2012) Si bien lxs trabajadores de salud mental también se consideran trabajadores de APS, se piensa como un continuo que va desde el abordaje más generalista al más especializado. Al delegar estas funciones lxs TCST pueden sentirse más habilitadxs para integrar el aspecto de la salud mental en su trabajo diario en las visitas domiciliarias, favoreciendo la accesibilidad y continuidad de cuidados.

Si las capacidades expresivas, perceptivas y creativas dan cuenta de indicadores de salud, la escucha de las dificultades expresivas y las dificultades para conectarse con la experiencia musical, se correspondían muchas veces con vivencias de situaciones problemáticas. En estos casos, fueron abordados en el momento, brindando contención o bien, derivados a otrxs profesionales o dispositivos según el caso lo requiriera. Esto permitió también pensar futuros abordajes y armar proyectos con otrxs trabajadores del Hospital. (Se realizó un proyecto de taller vincular entre mamás y bebés en conjunto con la Terapista Ocupacional y propuestas para el abordaje de la problemática del suicidio con lxs TCST)

En las visitas realizadas con lxs TCST a los parajes de Lago Rosario y Los Cipreses, la experiencia musical permitió el acercamiento a aspectos culturales, históricos, espirituales, de las personas, habilitando muchas veces el recibimiento de las personas y una mayor apertura al diálogo. Se registraron personas que se vinculaban a lo musical desde la ejecución instrumental y composición de canciones, así como también a la fabricación de instrumentos musicales. Se considera que los aspectos creativos resultan propiciadores de salud, ya que la creatividad permite adaptarse a situaciones y encontrar nuevas formas de resolver problemas (Pellizari y Rodríguez, 2005).

En uno de los parajes visitados, las experiencias musicales permitieron el acceso a cuestiones del legado cultural mapuche (instrumentos, historias en relación a celebraciones y a la lengua mapuche) en continua tensión entre la pérdida, la recuperación, la transformación, fuertemente relacionados con historias familiares. La historia de desalojos y pérdida de patrimonio cultural (lengua, festividades, medicina ancestral), la falta de ofertas de trabajo remunerado y de opciones de actividades recreativas y culturales que pudieran resultar en un aporte al sentido de existencia, la hostilidad climática y de acceso a recursos, la historia de colonización, son algunos de los aspectos mencionados por las personas visitadas como aspectos de mayor dificultad. Otras de las características mencionadas por lxs pobladores fueron la tranquilidad, la ayuda mutua entre vecinxs y la conexión con la naturaleza, como aspectos favorecedores de la salud. Las canciones y los instrumentos mapuches, permitieron conocer la problemática de la pérdida de celebraciones como el "Camaruco del lago", lengua y costumbres del pueblo. Por motivos de la duración temporal de la rotación no fue posible hacer un trabajo de recuperación o elaboración de estos aspectos, pero se espera que al realizar una "devolución" al equipo de salud mental del Hospital, se puedan abordar estas temáticas.

Otra de las formas de conocer y trabajar con la comunidad fue la experiencia de consultorio compartido con otrxs profesionales (terapia ocupacional, psicología, pediatría, fonoaudiología). La práctica de compartir consultorio con pediatría fue útil como herramienta para integrar la salud mental en la estrategia de Atención Primaria de la Salud. Se pudo trabajar en intervenciones focales (aprovechando la visita a lx pediatra como oportunidad para la intervención) generando un espacio dentro de la consulta para favorecer las experiencias sonoro-musicales entre niñxs y xadres. Las consultas de pediatría se generaban tanto para el "control del niñx sanx" (se toman parámetros como el peso, la altura, vacunación, etc.) como para consultas por demanda espontánea por distintas circunstancias de salud.

Se observó cierta dificultad para los intercambios cuerpo a cuerpo desde la sonoridad, la corporalidad y la gestualidad. Algunxs referentes adultxs (madre, padres) mencionaron no cantarle a lxs niñxs o exponerlos a música editada o pregrabada en mecedoras, no poder cantarle o conectarse emocionalmente con lxs niñxs. Se considera que los intercambios con la persona adulta son fundantes de la subjetividad humana y son 
imprescindibles para el desarrollo (Giacobone, 2009). Como hipótesis se podía considerar que esta dificultad podría estar relacionada con un rasgo de la época, la propia experiencia de vida de lxs adultxs, así como también por las preocupaciones de éstos por problemas económico-sociales o la incertidumbre misma de la pandemia por Covid con todas sus consecuencias en el tejido social y económico. Es por esto que se consideró prioritario trabajar en la prevención de posibles problemáticas de salud mental a partir de la promoción del vínculo saludable entre cuidadores adultxs y bebés.

A partir de la observación de ciertas dificultades en algunxs referentes adultxs para vincularse con lxs niñxs, se ha trabajado de forma conjunta con lxs TCST para que puedan continuar realizando visitas a los domicilios y brindar así mayor acompañamiento en este momento tan significativo para el fortalecimiento de la salud mental y el desarrollo de lxs niñxs.

La inclusión de la experiencia musical con niñxs en el consultorio de pediatría permitió brindar un espacio gratificante y lúdico en la consulta así como observar aspectos del desarrollo saludable en niñxs observando la vinculación con objetos, con otras personas, la exploración, la organización de discursos.

Otras de las intervenciones preventivas realizadas en conjunto con el equipo de salud fueron la realización de un jingle preventivo para el día de prevención del HIV, con una estructura de una canción que lxs TCST reconocían como una de las "más escuchadas" en la comunidad. Se realizó un cambio de letra para poder transmitir información en relación a los cuidados, trabajando grupalmente en el equipo de TCST en la ejecución instrumental y vocal. El mismo fue grabado y difundido en la radio comunitaria. Se realizaron también encuentros con la primera línea de intervención de COVID 19 a fin de aportar en el reconocimiento de estrategias personales y grupales de afrontamiento al estrés a fin de favorecer en la disminución de factores que pudieran estar resultando en un mayor padecimiento. Estas intervenciones permitieron conectar a lxs trabajadorxs de salud con aspectos creativos y lúdicos resultando un aspecto de promoción de su propia salud mental.

\section{Conclusiones}

Este escrito intentó dar cuenta del trabajo en prevención y promoción de salud realizado en la rotación de cuatro meses por un hospital rural. Quedaron fuera de este trabajo, otras intervenciones pensadas desde la lógica de la atención primaria, enfocadas en aspectos clínicos o de rehabilitación.

La musicoterapia con perspectiva comunitaria permitió ampliar los conceptos y prácticas sobre participación de las personas en su proceso de salud, favoreciendo la accesibilidad y el aprovechamiento de oportunidades para la intervención preventiva en salud mental.

A su vez, la música como producto cultural, permite un acercamiento desde el sistema de salud con experiencias culturalmente aceptables. Para esto es necesario tener en cuenta los aspectos de la interculturalidad, ya que se corre el riesgo de perpetrar lógicas coloniales. La pregunta por la propia cultura y la pregunta por la otredad deben estar siempre presente en este tipo de trabajo comunitario.

Es posible afirmar que la intervención en visita domiciliaria junto con TCST permite a las personas visitadas: mostrarse más permeables al diálogo y recibimiento del equipo de salud, ampliar las problemáticas abordadas desde salud pública, reconocer aspectos creativos expresivos en las personas, conocer factores sociohistóricos e identitarios de los pueblos, realizar intervenciones focales que propicien aspectos saludables, pesquisar factores de riesgo asociados a la salud mental y a partir de allí efectuar las derivaciones, capacitar, habilitar y delegar funciones de promoción de salud mental en lxs TCST, incluir el aspecto estético en la consideración de determinantes de salud dentro del equipo de trabajo.

La intervención en consultorio compartido con pediatría posibilitó un espacio más placentero para las consultas, la pesquisa de situaciones de dificultad a nivel vincular con referentes adultxs y una posibilidad más de escucha de la expresividad de lxs niñxs teniendo en cuenta su derecho a ser escuchadxs (Ley 26061, 2005) 
La contribución de la musicoterapia amplía la visión de salud positiva entendiendo a los aspectos expresivos, perceptivos, estéticos y creativos como parte de la construcción de la misma.

\section{ReFERENCIAS}

Argandoña, M. (2012). Integración de cuidados de salud mental en la atención primaria de la salud. Universidad Nacional de Lanús.

Carrizo, L. (2020). Aportes desde una perspectiva estética de la Musicoterapia a producción artística en la Empresa Social “Quenuir". ECOS -Revista Cientifica De Musicoterapia Y Disciplinas Afines, 5(1), 17-29. Recuperado a partir de https://revistas.unlp.edu.ar/ECOS/article/view/10400

Dirección General de Estadística y Censos, Proyecciones y Estimaciones, (s. f.) Población estimaciones por gobierno local, Provincia de Chubut, años 2011-2021 [Archivo Excel] https://estadistica.chubut.gov.ar/index.php?opti on $=$ com_content $\&$ view $=$ article\&id $=311 \&$ Itemid $=243$

Dirección Nacional de Salud Mental y Adicciones (2015) Autores Varios (2015) Políticas públicas de salud mental: enfoques, estrategias y experiencias con base en la comunidad. - 1a ed. - Ciudad Autónoma de Buenos Aires. Ministerio de Salud de la Nación.

Dirección Nacional de Salud Mental y Adicciones (2020a) Salud Mental y Apoyo Psicosocial en emergencias y desastres: Plan de Acción. Ministerio de Salud de la Nación Argentina. https://bancos.salud.gob.ar/recurso/sal ud-mental-y-apoyo-psicosocial-en-emergencias-y-desastres-plan-de-accion.

Dirección Nacional de Salud Mental y Adicciones (2020b) Recomendaciones para el abordaje territorial en salud mental en la pandemia por Covid 19 BUSCAR LINK

Dirección Nacional de Salud Mental y Adicciones (2021) Recomendaciones sobre salud mental para los equipos de salud. Actualización. En Ministerio de Salud de la Nación, Plataforma Virtual de Salud, https://plataformavirt ualdesalud.msal.gov.ar/course/view.php?id=343\$ion=3

Gauna, G. (2009). Diagnóstico y abordaje musicoterapéutico en la infancia y la niñez. Koyatún Editorial.

Giacobone, G. (2009) “La musicoterapia clínica en atención temprana del desarrollo infantil” en Gauna (2009) Diagnóstico y abordaje musicoterapéutico en la infancia y la niñez. Koyatún Editorial.

Ley 26.061 (2005) Protección Integral de Niños, Niñas y Adolescentes. Consultada en http://servicios.infoleg.gob.a r/infolegInternet/anexos/110000-114999/110778/norma.htm

Ley 26.657 (2010) Ley Derecho a la Protección de la Salud Mental http://servicios.infoleg.gob.ar/infolegInternet/a nexos/175000-179999/175977/norma.htm

Ministerio de Salud de la Provincia de Chubut (2016). "Programa Ocupacional Laboral”.

Organización Mundial de la Salud. Declaración de Alma Ata. Conferencia Internacional sobre Atención Primaria de Salud; 6-12 de junio 1978; Alma Ata, USSR. Ginebra: OMS; 1978.

Organización Panamericana de la Salud (1990) Declaración de Caracas. Descargada en https://paho.org/Declaracio n_de_Caracas

Organización Panamericana de la Salud. (2014). Prevención del Suicidio, un imperativo global.

Pazos Rios, R. y Grupo vocal Sur, 1989, Mi Pueblo del Molino [Canción]. En Sonetos para mi raza. https://youtub e.com/watch?v=rAd3qvzyBhs

Pellizari, P., y Rodríguez, E. (2005). Salud, Escuch a y Creatividad. Ediciones de la Universidad del Salvador.

Pellizari, P. (2011). Crear Salud. Patricia Pellizari Editora

Weinstein, L. (2011). Salud y Autogestión. Editorial Tierra del Sur. 